\title{
Ultrasonography of a cervical pregnancy
}

Author: Jesper Agrell

Correspondence: jesper.agrell @lvn.se

\section{Suggested citation format:}

Agrell, Jesper (2014). "Ultrasonography of a cervical pregnancy". Wikiversity Journal of Medicine 1 (2). doi:10.15347/wjm/2014.011. ISSN 20018762 .

First submitted: 15 November 2014

Accepted: 15 November 2014

Last updated: 10 April 2016

\section{Licensing:}

This is an open access article distributed under the terms of the Creative Commons Attribution-

ShareAlike License, which permits unrestricted use, distribution, and reproduction in any medium, provided the original author and source are credited.

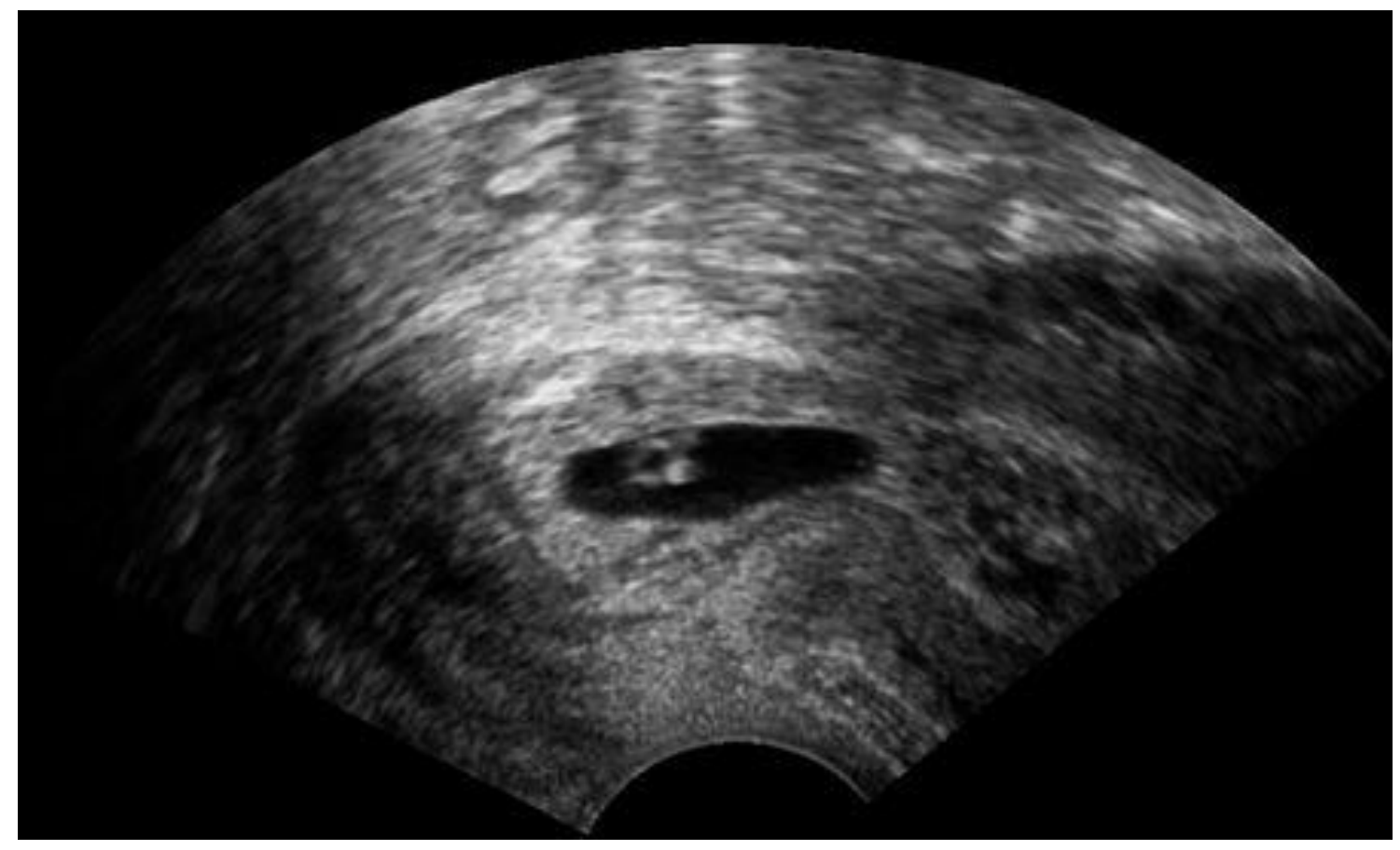


A woman in her 20s came to the gynecologic clinic because of a positive pregnancy test and a history of a previousectopic pregnancy. The vaginal ultrasonography performed by Dr. Jesper Agrell showed a gestational sac in the cervixas displayed in the sagittal plane. The corpus of the uterus is located at right in the image. There was a discernible heartbeat, and the gestational sac diameter corresponded to a gestational age of 5 weeks. The distance from the gestational sac to the external orifice was only 15 millimeters.

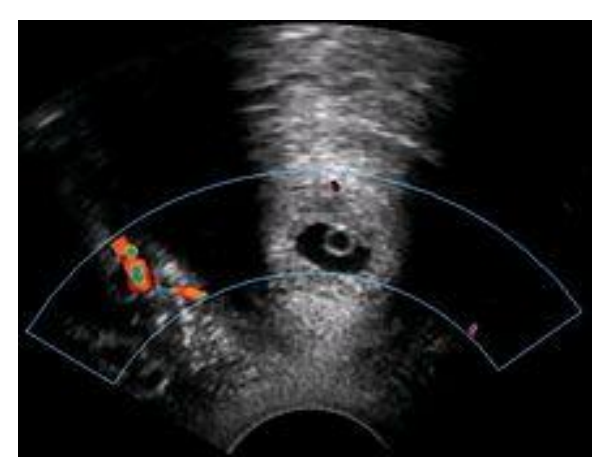

Trans-section of the cervix with Doppler ultrasonography, showing that the pregnancy (center) is at the same level as the uterine artery (giving a colored pattern to the right on doppler). 